\title{
Plausible fast \& economic detection of SARS-CoV infection using paper-based isoelectric focusing: a hypothesis
}

\author{
Arindam Kushagra \\ Amity Institute of Nanotechnology, Amity University Kolkata, INDIA. PIN: 700135 \\ email: arindam.kushagra@gmail.com
}

\begin{abstract}
In the wake of current pandemic of COVID-19, there has arisen an urgent need to come up with novel ways to detect the infected person with SARS-CoV-2 at an extremely fast pace so that the person is immediately quarantined to prevent further transmission of the virus to other susceptible individuals. Such quick detection measures would allow the timely mitigation of the dreadful disease which has claimed close to 100,000 lives worldwide. In this article, a very fast and cheap way to detect the presence of SARS-CoV in the biopsied tissue has been proposed. The current method discussed here would drastically reduce the time of detection of an infected person to less than $\sim 6$ hours in addition to the economic benefit of using paper-based isoelectric focusing. The infecting coronavirus that causes severe respiratory distress contains four major types of structural proteins: spike (S) proteins, membrane $(\mathrm{M})$ proteins, envelope $(\mathrm{E})$ proteins \& nucleocapsid $(\mathrm{N})$ proteins. Peculiar isoelectric points of these four structural proteins have been proposed to detect the SARS$\mathrm{CoV}$ infection. This work would be of immense interest to the scientists, researchers as well as health professionals who are trying to mitigate the spread of the disease as well as to cure it at the same time.
\end{abstract}

\section{Introduction}

With the spread of the fatal COVID-19 that has become a pandemic, researchers worldwide are trying to accelerate the detection as well as treatment regimens of the zoonotic coronavirus-related disease. The disease is caused by a family of virus, known as Coronavirus, causing the acute respiratory distress in the patients. Thus, the disease that is caused by such virus is known as Severe Acute Respiratory Syndrome abbreviated as SARS. This virus is known to contain four major structural proteins, spike (S) proteins, membrane 
(M) proteins, envelope $(\mathrm{E})$ proteins \& nucleocapsid $(\mathrm{N})$ proteins that are spatially arranged and distributed within the spherical virus particle, as shown in the schematic in Fig. 1.

31

\begin{tabular}{|c|c|}
\hline Structural protein of SARS-CoV & Isoelectric point $(\boldsymbol{p} \boldsymbol{I})$ \\
\hline Spike (S) & 5.4 \\
\hline Envelope (E) & 6.3 \\
\hline Membrane (M) & 9.8 \\
\hline Nucleocapsid (N) & 10.5 \\
\hline
\end{tabular}

Fig. 1 Schematic diagram of the SARS-CoV is shown, depicting the spatial arrangement of various structural proteins [1].

The pathogenicity of the virus depends on its attachment with the human angiotensinconverting-enzyme-2 (ACE-2) via S-protein. Thus, the S-protein has garnered great attention of the scientific community to deter the infection and to find its cure [2]. The four major structural proteins namely, S, E, M \& N of the SARS-CoV have distinct isoelectric points $(p I)$ ranging from acidic to basic $\mathrm{pH}$ ranges, as shown in Table-1.

Table 1 Isoelectric points $(p I)$ of major structural proteins of SARS-CoV are shown, depicting a spike in $p I$ values of $\mathrm{M} \& \mathrm{~N}$ proteins in the alkaline range [3].
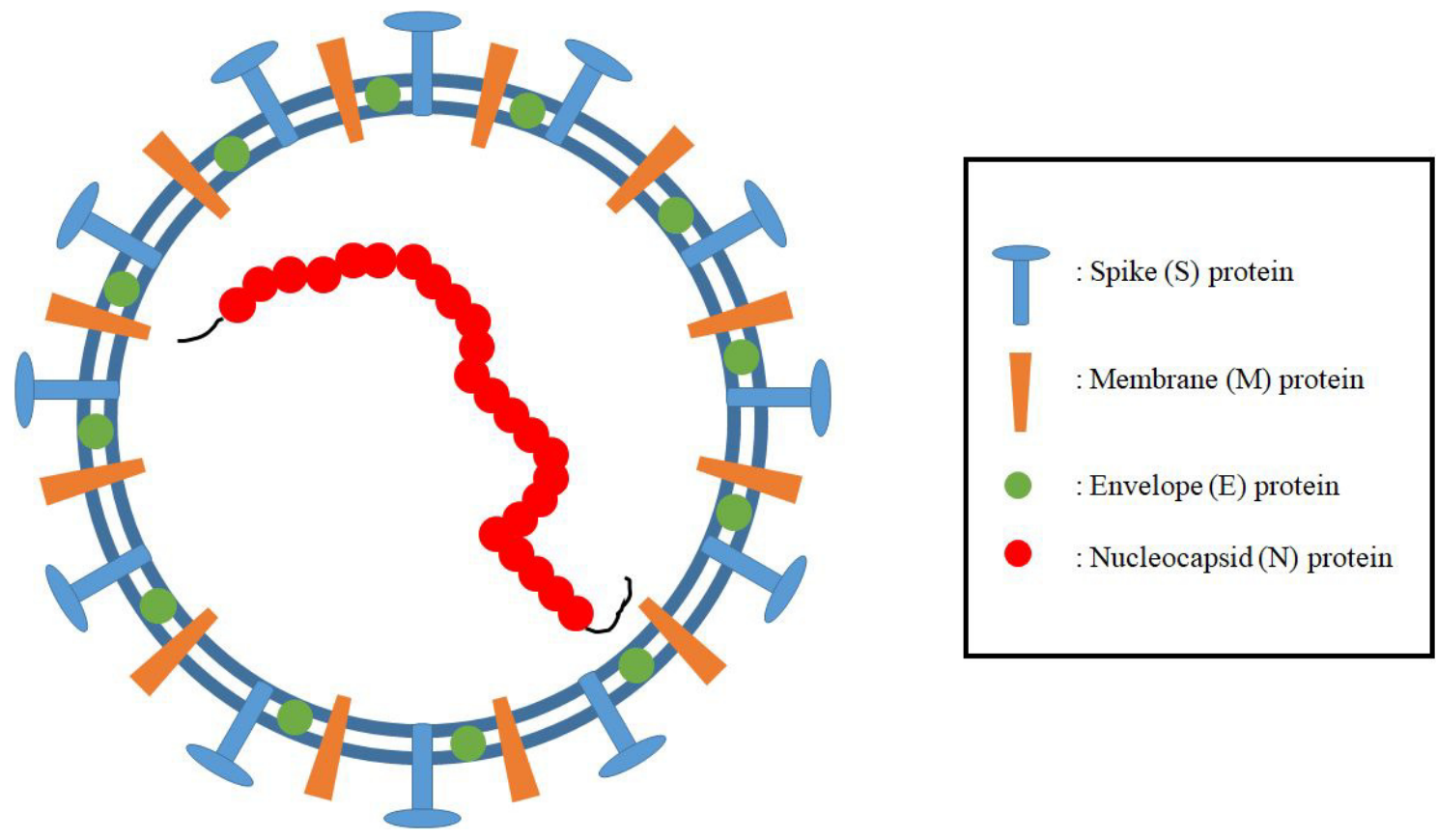
41 Currently, the detection of infected person is done by the FDA approved RT-PCR test that requires specialized costly instruments to perform polymerase chain reaction (PCR) using a reverse transcriptase (RT) and specific DNA primers to detect the RNA from infecting coronavirus [4]. In this article, the concept of paper-based isoelectric focusing is proposed to identify the presence of SARS-CoV infection in the sample using the isoelectric points of M $\& \mathrm{~N}$ structural proteins that fall in the alkaline range ( $p I$ values: $9.8 \& 10.5$ ). Such choice of proteins to detect SARS-CoV is made because all the other proteins present in the extracted sample from the patient would not normally have as high isoelectric points as that of structural proteins $M \& N$. Nearly $10-15 \%$ proteomes from the non-redundant NCBI protein database (having 86 million proteins) \& $~ 5 \%$ proteomes from the UniProtKB/TrEMBL protein database (having 63 million proteins) have $p I$ values $\sim 10$ [5]. Viral characterizations have been reported earlier by the analyses of capsid proteins [6, 7]. Here, a very fast and economically viable way to detect the infection of the sample with SARS-CoV has been proposed which would pave the way to reduce the detection timespan that could be used in low-cost settings in developing countries.

\section{Methodology}

$\underline{\text { Virus purification from cell lysate [8] }}$

- Sample containing the virus would be subjected to hypotonic burst using $5 \mathrm{ml}$ of double distilled water following 15 minutes incubation at room temperature. The cell debris would be separated from the infecting virus particles by centrifuging the lysate at $3000 \times \mathrm{g}$ for 15 minutes. The resulting supernatant would be containing the virus particles that would further be removed by pipeting and mixing with equal volumes of $2 \times$ DMEM containing 4\% FCS, $2 \%$ penicillin-streptomycin solution, and $4 \mathrm{mM} \mathrm{L}-$ glutamine for restoration of the tonicity as in case of the original virus stock.

- Alternatively, viruses can also be collected from the breath samples of the suspected individuals infected with coronavirus [9]. The virus samples could be collected using simple breath-analyzer tools that are regularly used by law-enforcing agencies to check drunk driving cases.

\section{Separation of viral capsid proteins from viruses [10]}

- Virus particles have been reported to degrade at $\mathrm{pH}$ 10.5. Using the mentioned protocol in the reference therein, the viral capsid proteins would be separated for further analytical procedures. 


\section{Paper-based isoelectric focusing [11]}

- The setup for paper-based would be prepared using the materials \& methods described in the reference therein. The separation of viral capsid proteins would take $\sim 2$ hours if the applied focusing voltage was kept at $500 \mathrm{~V}$ whereas the time of $p I-$ based separation would get reduced to $\sim 30$ minutes upon application of focusing voltage of $1500 \mathrm{~V}$. The schematic diagram for such analytical procedure is shown in Fig. 2.

TOP VIEW

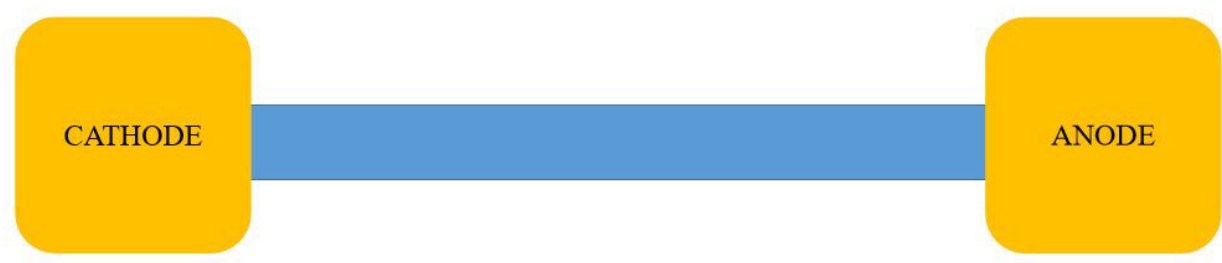

SIDE VIEW

\section{Discussion}

The above discussed method to analyze the sample collected from the diseased person for SARS-CoV infection provides a novel way to detect such infections. This analytical tool is extremely time-efficient (takes less than $\sim 6$ hours) and cost-effective in comparison to currently employed techniques that require DNA polymerases, specific DNA primers that would complement the genetic sequence of the infecting virus. In the proposed method, the viral capsid proteins could be detected just on the basis of their respective isoelectric points with $p I$ values of $\mathrm{M} \& \mathrm{~N}$ structural proteins in the very peculiar range $(p I>9)$. This range is extremely uncommon in common histological samples, thus would become a unique 
signature for the presence of SARS-CoV in the analyzed sample. This work would be of immense interest to the scientists, researchers as well as health professionals who are working towards the mitigation of the dreadful respiratory viruses, especially in the wake of current pandemic COVID-19.

\section{References}

[1].J.S. Peiris, Y. Guan, K.Y. Yuen, Severe Acute Respiratory Syndrome, Nat Med. 2004 Dec;10(12 Suppl):S88-97.

[2].L. Du, Y. He, Y. Zhou, S. Liu, B-J Zheng, S. Jiang, The spike protein of SARS-CoV-a target for vaccine and therapeutic development, Nat Rev Microbiol. 2009 Mar;7(3):226-36.

[3].S. Liu, T. Guo, X. Ji, Z. Sun, Bioinformatical study on the proteomics and evolution of SARS-CoV, Chin Sci Bull. 2003;48(13):1277-1287.

[4].COVID-19 RT-PCR test (LabCorp Laboratory Test Number: 139900), https://www.fda.gov/media/136151/download, accessed on $13^{\text {th }}$ April, 2020.

[5]. L.P. Kozlowski, Proteome-pI: proteome isoelectric point database, Nucleic Acids Res. 2017 Jan 4;45(D1):D1112-D1116.

[6].J.J. Thomas, B. Falk, C. Fenselau, Viral Characterization by Direct Analysis of Capsid Proteins, Anal Chem. 1998 Sep 15;70(18):3863-7.

[7].D.O. Cliver, Capsid and Infectivity in Virus Detection, Food Environ Virol. 2009 Dec;1(3-4):123-128.

[8].B.W. Kong, L.K. Foster, D.N. Foster, A method for the rapid isolation of virus from cultured cells, Biotechniques. 2008 Jan;44(1):97-9.

[9].N.H.L. Leung et al., Respiratory virus shedding in exhaled breath and efficacy of face masks, Nat. Med. 2020. doi: https://doi.org/10.1038/s41591-020-0843-2

[10]. F.A. Anderer, H.D. Schlumberger, M.A. Koch, H. Frank, H.J. Eggers, Structure of simian virus 40 II. Symmetry and components of the virus particle, Virology. 1967 Jul;32(3):511-23.

[11]. S. Yu, C. Yan, X. Hu, B. He, Y. Jiang, Q. He, Isoelectric focusing on microfluidic paper-based chips, Anal Bioanal Chem. 2019 Aug;411(21):5415-5422. 